\title{
Information Modelling on Mechanized Earthworks
}

\author{
Shigeomi Nishigaki $^{a}$, Katsutoshi Saibara ${ }^{b}$, and Shigeo Kitahara ${ }^{c}$ \\ ${ }^{a}$ Mazaran, Co., Ltd., and Kick, Co., Ltd., Japan \\ ${ }^{b}$ Kick, Co., Ltd., Japan \\ ${ }^{c}$ Kumagaigumi, Co., Ltd., Japan \\ E-mail: sleepingbear@c2m.com, saibara@c2mp.com, skitahar@ku.kumagaigumi.co.jp
}

\begin{abstract}
-
Mechanized earthworks for post-disaster reconstruction largely forks into unmanned construction at hazardous working sites and manned one at less hazardous sites. In order to avoid secondary disasters, it is significant and required to compensate operator's realistic sensation in order to enhance spatial awareness in the mechanized earthworks. The mechanized earthworks consist of a series of discrete works, which are sometimes independently and at other time interactively performed by different workers and managed by small and medium contractors. Generally, a variety of construction machines made by different manufactures are used there. On the other hand, diverse machine guidance systems come from different vendors. Most of them are self-contained and constitute an independent unit in and of itself. Here, are demanded inexpensive and easy-to-use software systems with sensors capable of easily mounting and demounting. The software systems of those should be not embedded in specific construction machines but available to multiple ones. To overcoming problems like those, our primary efforts target on fabricating information modelling system for mechanized earthworks by utilizing open source software. The information modelling here means a process involving acquisition of field data and feedback of relevant digital representations of physical and functional characteristics of works in progress. This paper presents framework of field data acquisition and exploratory spatio-temporal visualizations, enhancement of spatial awareness, enhancement of communication, and conclusions with a discussion of the contributions included in this paper. Finally, some open problems are also pointed out.
\end{abstract}

Keywords -

Information modelling; Mechanized earthworks; Unmanned construction; Realistic sensations;
Potential hazards; Exploratory spatio-temporal visualization; Enhancement of Communication

\section{Introduction}

Motivations and objectives in this study, and structure of this paper are reported in this Section.

\subsection{Compensation of Operator's Realistic Sensations}

Mechanized earthworks for post-disaster reconstruction largely forks into unmanned construction at hazardous working sites and manned one at less hazardous ones. When facing such dangerous situations, in order to avoid any secondary disasters, we demand unmanned operations.

Handling joysticks to operate construction machines, either directly or remotely, is an inherently eye-hand coordination task. The eye-hand coordination means to control eye movements with hand movements, and as processing visual representation of the situational views to handle joysticks along with the use of proprioception of the hands, or vice versa

In case of manned operation at less hazardous working site for loading, hauling and dumping (LHD) of earth and rocks, operators would handle their machines based on their own realistic sensation given on the seat of their pants. For examples, distance and clearance perception based on front views, possible motion space, and velocity, acceleration, jerk, jolting, rattling, rolling, pitching, and yawing of the machine body.

In case of unmanned operation at hazardous working sites for removal of earth and rocks, operators can't feel their realistic sensation, and have to remotely operate their machines in a narrow field of view from cameramonitor system at the control station. Problems here include:

(1) Machine operability,

(2) Difficulty in a task at hand,

(3) Performances limited by bearings of operators in 
their behavioural frameworks, and

(4) Continuous/intermittent mental workload on them.

Therefore, it is significant and required to compensate operator's realistic sensation in order to enhance spatial awareness, in other words, conscious awareness of actions.

\subsection{Enhancement of Communication}

Mechanized earthworks consist of a series of discrete works, e.g., digging, loading, hauling, dumping, grading, embanking, compacting, etc., which are sometimes independently and at other time interactively performed by different workers and managed by small and medium contractors. Generally, a variety of new and used construction machines made by different manufactures are utilized in the mechanized earthworks.

It is said that machine guidance systems will be able to improve construction accuracy and productivity [1], [2]. At present, diverse machine guidance systems come from different vendors. Most of them are self-contained and constitute an independent unit in and of itself. Considering use of different construction machines, it is difficult for workers to coordinate data among the diverse machine guidance systems, and to generate significant information based on the data as linking them to the other systems. In addition, the up-front cost of the machine guidance systems might be a huge burden and a ball and chain to the small and medium contractors in Japan. They would demand inexpensive and easy-to-use software systems with devices, which are capable of easily mounting and demounting on different construction machines made by various manufactures. The software systems of those should be not embedded in specific construction machines but available to multiple ones.

Therefore, it might be difficult for workers to grasp current conditions of the each work related to one another, and, not to mention, almost impossible for them to know or reason the present and future situations of the whole work process on a real time basis. Here, it is very important to enhance communication as to work process and operations between the people concerned.

\subsection{Objectives}

To overcoming the problems like these as described above, our primary efforts target on fabricating an information modelling system on mechanized earthworks by utilizing open source software based on GNU General Public License [3]. The information modelling here means a process involving acquisition of field data and feedback of relevant digital representations of physical and functional characteristics of works in progress.

\subsection{Structure of this Paper}

This paper is organized as follows. First, we introduce framework of field data acquisition and exploratory spatio-temporal visualizations in Section 2. Secondly, in Section 3, we report enhancement of spatial awareness, put concretely, feedback of quantitative indexes and exploratory spatio-temporal visualizations of potential hazards in unmanned construction. Thirdly, enhancement of communication in the mechanized earthworks is given in Section 4. In Section 5, we conclude with a discussion of the contributions included in this paper. Finally, some open problems are also pointed out.

\section{Framework of Field Data Acquisition and Information Feedback}

Discrete spatio-temporal events are occurred in space with time by construction work practices. Data pertaining to the events are automatically collected in real time by the inexpensive devices. These devices are capable of easily mounting and demounting, which consist of six-degree accelerometers, GPS receivers, active tags, compact PCs, smart phones, etc., and sometimes manually acquired by key-in as needed.

Figure 1 shows framework to acquire filed data and feedback relevant digital representations of physical and functional characteristics of works in progress.

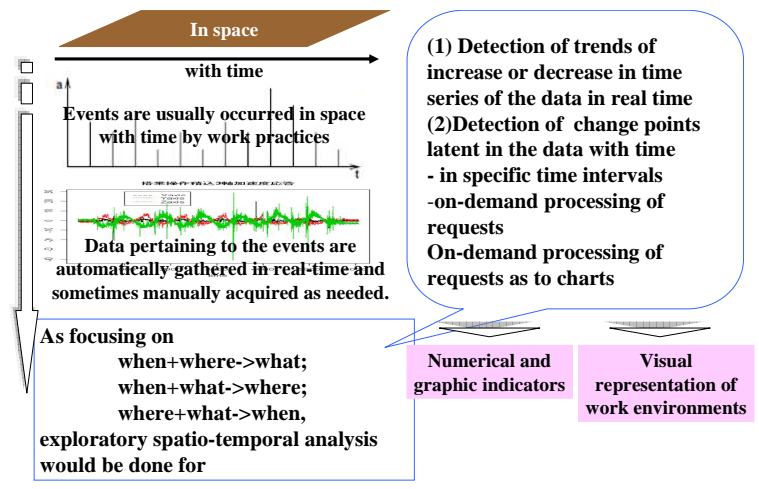

Figure 1. Framework of filed data acquisition and information feedback

Changes over time in spatio-temporal events are classified as follows [4], [5]:

(1) Existential changes, i.e. appearance and disappearance,

(2) Changes of spatial properties: location, shape or/and size, orientation, altitude, height, gradient and volume, and

(3) Changes of thematic properties expressed through 
values of attributes: qualitative changes and changes of ordinal or numeric characteristic.

Exploratory spatio-temporal visualization of the above changes would be done by the open source software the $\mathrm{R}$, which is a language and environment for statistical computing and graphics [4], [6] as focusing on:

(1) When and where, then what,

(2) When and what, then where, and

(3) Where and what, then when,

Figure 2 shows an image of the exploratory spatiotemporal visualization in this study. Numerical and graphic indicators here would be derived by:

(1) Detecting trends of consecutive increasing or decreasing values and change points latent in time series of the data, and

(2) Providing visual representation of work environments based on-demand processing of requests.

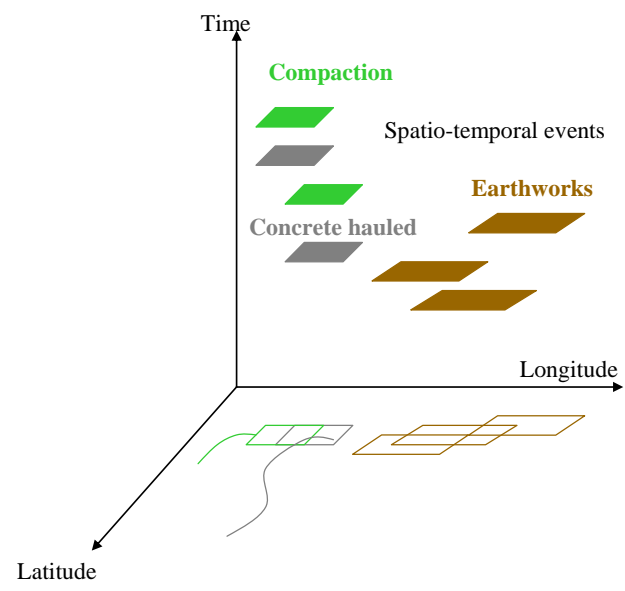

Figure 2. Image of the exploratory spatio-temporal visualization in this study

\section{Enhancement of Spatial Awareness}

In this Section, we present feedback of quantitative indexes and exploratory spatio-temporal visualization that will be able to enhance operator's spatial awareness.

\subsection{Feedback of Quantitative Indexes}

The spatial awareness is very important for operators at their control room to infer and understand the present and future surroundings of their own construction machines or heavy-duty dump trucks and to watch themselves in the work space. In addition, it is significant and required to compensate operator's realistic sensation, when remotely operating construction machine at dangerous spots, for example, at the steep slope, at the ridge of steep cliff, etc.

Figure 3 shows the information flow to assist operators. Construction machines have on-board devices that are composed of tools as follows:

(1) Tri-axial accelerometer,

(2) Tri-angular velocity meter,

(3) GPS receiver,

(4) Smart Phone or tablet PC, and

(5) Communication module.

These devices would be inexpensive and capable of mounting and demounting on any construction machine for earthworks.

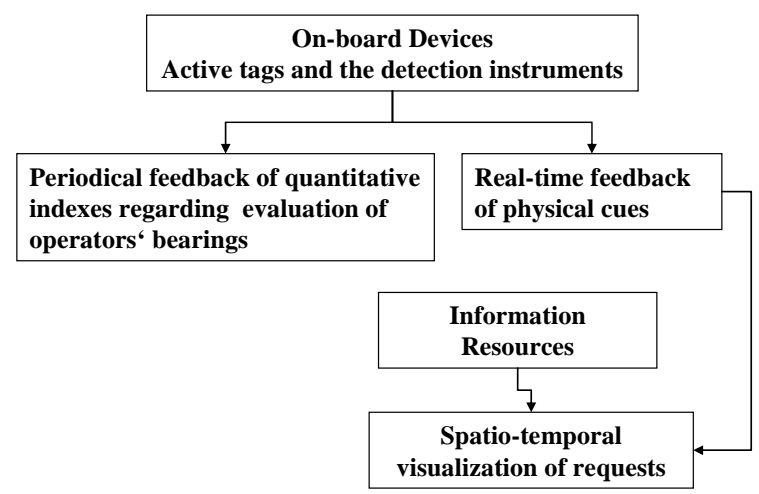

Figure 3. Information flow to assist operators

Besides, are used wireless LAN-based devices that consist of active tags and the detection instruments with about $100 \mathrm{~m}$ effective range [7]. The each dump truck carries an active tag. The detection instruments are put in position on the side of the entrance to the loading and the dumping spots, respectively. The detection instruments successively receive signals from the active tags with the dump trucks that come into the effective cover range. Then the detection instruments automatically send the Web server system via the Internet the time stamps and the IDs of the active tags.

Based on field data given by the above on-board devices, are periodically and automatically generated and feedback quantitative indexes regarding operators' bearings [8], the productivity [9], [10], and the physical cues [11].

Examples of the quantitative indexes as to operators' bearing include:

(1) Mean cross rate,

(2) Magnitude of impact loads,

(3) Operability of orientation control,

(4) Learning capability,

(5) Difficulty in a task at a hand, and

(6) Response capability

Examples of the quantity indexes related to productivity include: 
(1) Moving distance,

(2) Number of repositions,

(3) Actual operation rate, and

(4) Productivities of the LHD such as number of loading and dumping, distance hauled, work volume hauled, cycle time, etc.

Physical cues pertaining to posture of machine body and hazard factors latent in work places are feed backed in real time. Examples of the physical cues include:

(1) Posture of machine body such as inclination, rolling, pitching,

(2) Positions of bucket pin,

(3) Advance alert as to skidding, bumping, and rolling over, and

(4) Advance proximity warning

To let operators recognize danger efficiently when remotely operating their machines, the numeric and graphic indicators with respect to the quantitative indexes would be intuitively context-sensitive assists to compensate operators' realistic sensation.

Even under direct viewing, operators and the people concerned could not accurate at making inferences about the relative position of objects, the productivity of work in progress, and operators' bearings. For securing safety, reliable and effective remote control, it becomes very important to provide operators with the information described in this Section.

\subsection{Exploratory Spatio-temporal Visualization of Potential Hazards}

Since operators could not easily envisage any hazard latent in unmanned earthwork process without any back ground information in a narrow field of view from camera-monitor system at the control station. It would be helpful and significant for operators in the control station to see severe dangerous spots with marks, and to watch the advance proximity warning on their monitors, when approaching to them.

Besides, exploratory spatio-temporal visualization of terrain and hazards are given with information resources such as digital elevation model and digital maps. Examples of the exploratory spatio-temporal visualization include:

(1) Scatter plot,

(2) Ground surface contour,

(3) Heat map,

(4) Slope and aspect,

(5) Marginal plot of terrain, and

(6) Hazard map

Figure 4 shows the ground surface contour of construction area ant the trajectory of the heavy-duty dump truck (blue points), which hauls no-slump concrete to construct a multilayer check dam against avalanche of earth and rocks in Unzen-Fugendake. The multilayer check dam as shown in Figure 5 has been and is being constructed in severe hillside of which the gradients and aspects are shown in Table 1, and the gradients is visualized in Figure 6.

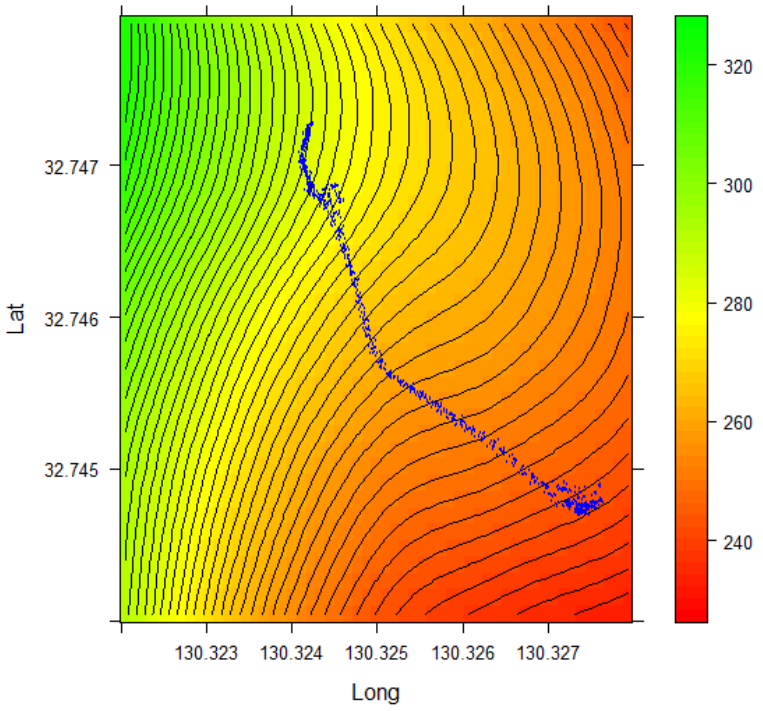

Figure 4. Ground surface contour and marginal plots of across each coordinate of construction area

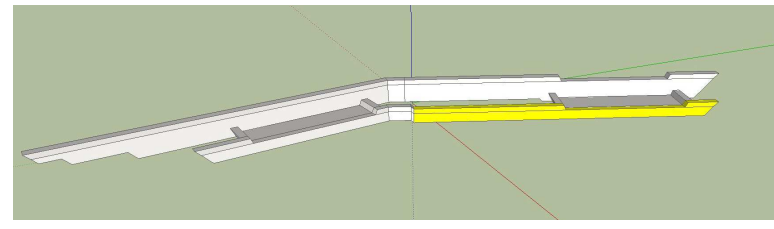

Figure 5. Multilayer check dam has been and is being constructed

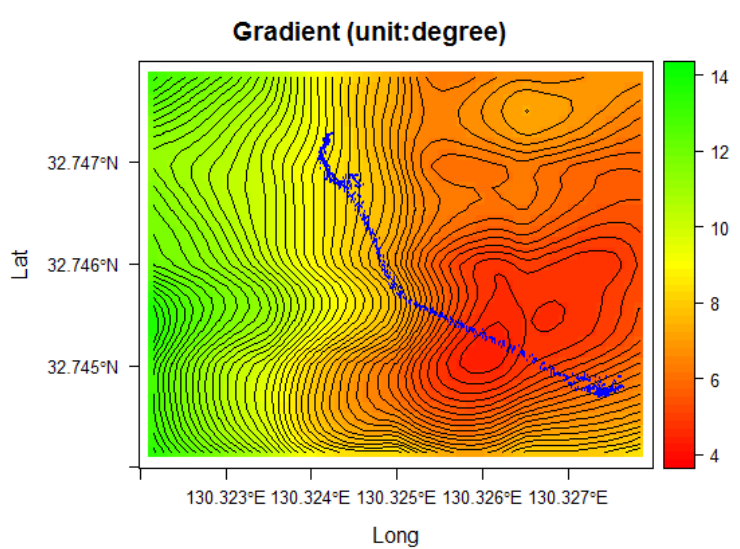

Figure 6. Visualization of gradients in construction area 
Figure 7 displays hazards regarding crosswise skiddy spots (red bubbles) latent in the no slump concrete haulage roads (blue dots). Also, we could visualize the longitudinal skiddy spots and the bumpy spots. For want of space, the charts like those are left out in this paper. The hazards latent in haulage road are detected based on existence of trends such as consecutive increasing or decreasing values in time series of tri-axial acceleration responses with longitudinal and latitudinal coordinates.

Table 1. Gradient and aspect (unit: degree)

\begin{tabular}{lrr}
\hline & Slope & \multicolumn{1}{c}{ Aspect } \\
\hline Min. & 4.289 & 47.530 \\
1st Qu & 6.034 & 103.680 \\
Median & 7.224 & 122.640 \\
Mean & 7.896 & 120.810 \\
3rd Qu. & 9.938 & 143.270 \\
Max. & 13.705 & 168.860 \\
\hline
\end{tabular}

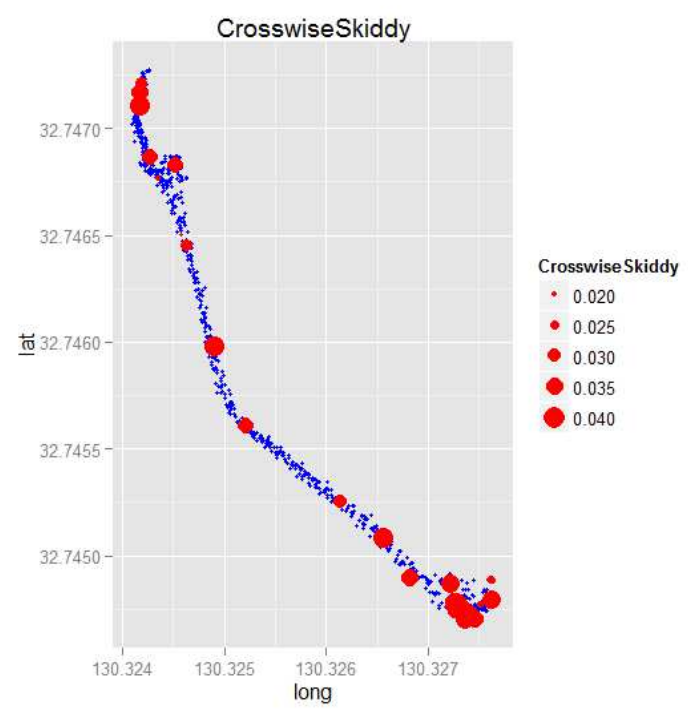

Figure 7. Sideways skiddy spots,

Hazardous spots like those might emerge at different places as the earthworks are progressing with time. To ensure safe and reliable haulage works, it is very significant to display the hazardous spots in advance on the monitor before starting and during work operations.

\section{Enhancement of Communications}

We have been and are developing the information modelling system, which enables the people concerned to share information on the no-slump concrete haulage and the compaction works in progress and to enhance communications among them. Figure 8 shows system configuration to enhance communications among the people concerned. This system enables them to peruse the analysis results, summary statistics and graphics of the works in progress anytime anywhere. Figure 9 shows a confirmation scene of compaction works in progress by the vibration roller in the outdoors other than at the control room.

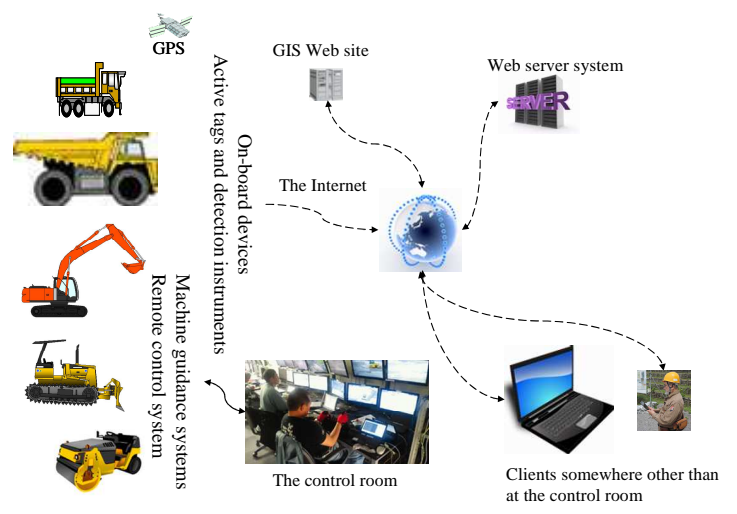

Figure 8. System configuration to enhance communication

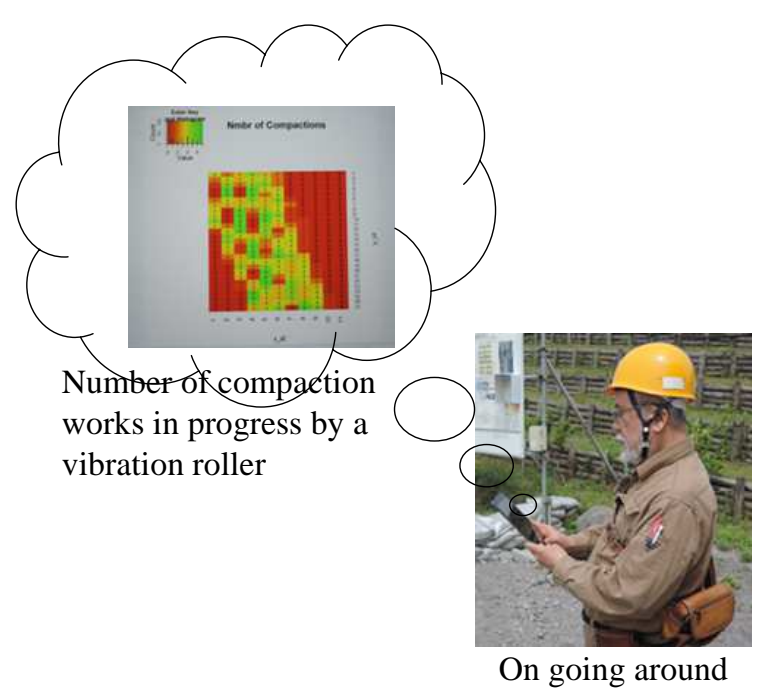

Figure 9. Example of confirmation of compaction works in progress by tablet $\mathrm{PC}$ in the outdoors

\subsection{Visualizations of No-slump Concrete Haulage Works}

The no-slump concrete is hauled by 10 ton dump trucks from the ready-mixed concrete batching plant to the spot where it is transferred to heavy-duty dump trucks. Subsequently, the heavy-duty dump trucks 
being remotely controlled haul the no-slump concrete to the designated dumping spot. In addition, a bulldozer spreads the no-slump concrete, and subsequently a vibration roller compacts [7].

Figure 10 shows the line chart of cumulative numbers both of arrivals of ton dump trucks to and the departures from the concrete batching plant for loading no-slump concretes.

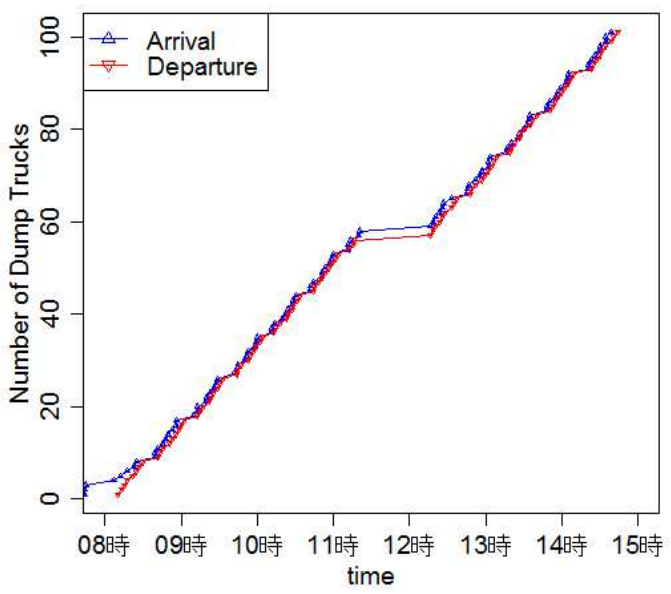

Figure 10. Line chart of arrivals and departures of 10 ton dump trucks at concrete batching plant

Figure 11 shows the progress curve of the no-slump concrete haulage.

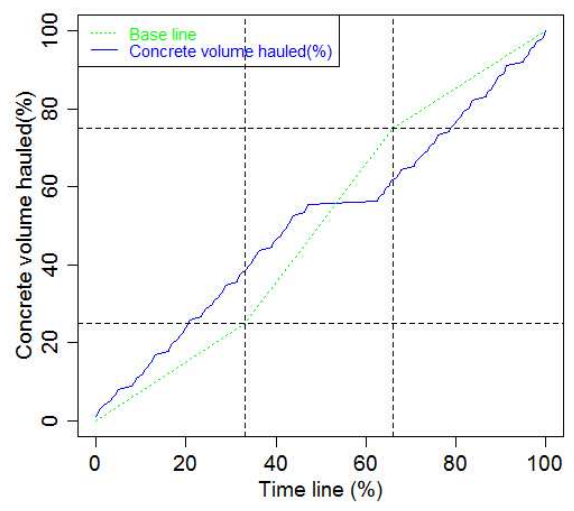

Figure 11. Progress curve of the no-slump concrete haulage

Besides, Figure 11 includes a base line for a comparison objective, which connects points of $0 \%$, $33 \%, 66 \%, 100 \%$ at the time line axis and the corresponding $0 \%, 25 \%, 75 \%, 100 \%$ at the axis of the concrete volume hauled with a green line.

Figure 12 shows a spatio-temporal visualization of movements of a heavy-duty dump truck being remotely controlled to haul the no-slump concrete toward the designated dumping spot. Then the heavy-duty dump truck runs at an average of 18 kilometres an hour.

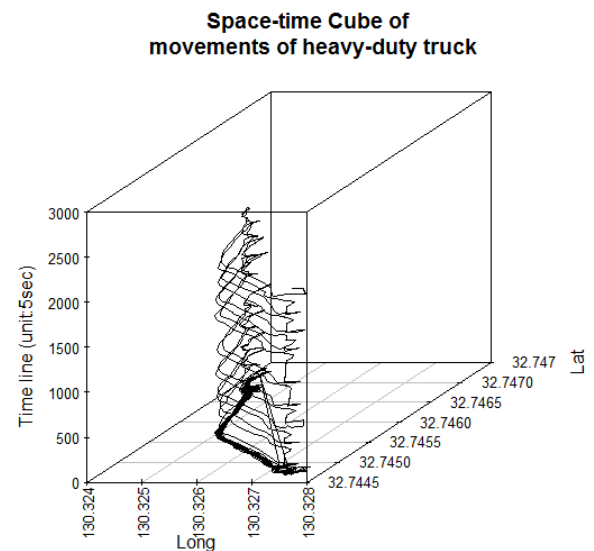

Figure 12. Movements of heavy-duty dump truck being remotely controlled

The people concerned can watch these Figures anytime, anywhere. It can be easily seen from these Figures that there are no queuing lengths at both of the ready-mixed concrete batching plant and the transhipment place to the heavy-duty dump tracks. In addition, there is nothing of accumulative delays in the movements of the heavy-duty dump trucks.

\subsection{Visualizations of Compaction Works}

Figure 13 shows an example of display of compaction works by the machine guidance system for the vibration roller at the control room. This machine guidance system is self-contained and constitute an independent unit in and of itself only for the operator at the control room. Therefore, the people except the operator can not watch the display somewhere other than at the control room.

In contrast, the information modelling system enables them to peruse analysis results, summary statics and exploratory spatio-tempral visualization of the compaction works in progress by the vibration roller anytime, anywhere.

Figure 14 shows the number of compactions in progress. The Information modelling system also provides data regarding work stating time, current time, work time elapsed, distance covered, and work finish 
time. Then the vibration roller walks at an average of 6.7 kilometres an hour.

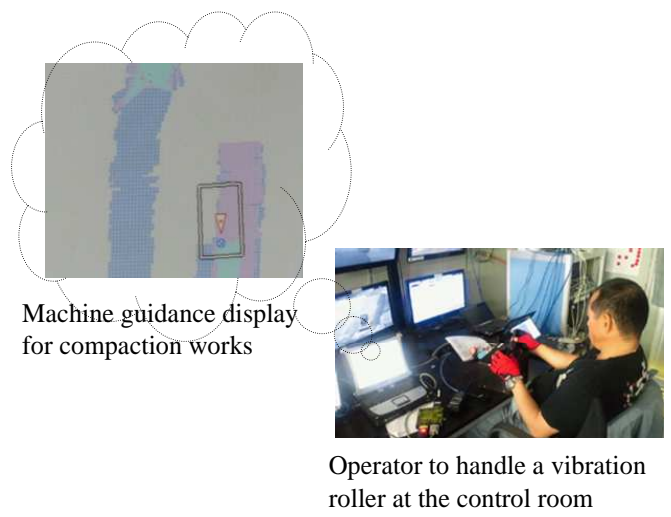

Figure 13. Display of compaction works by machine guidance system at control room
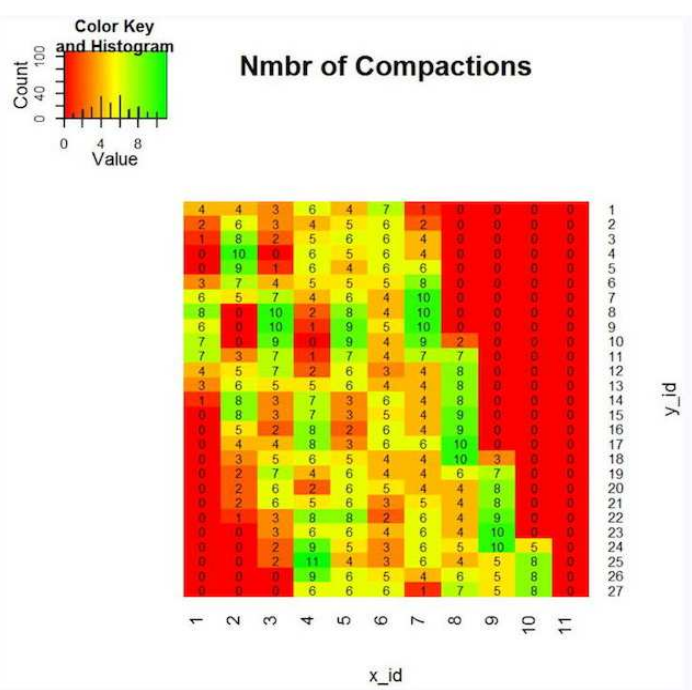

Figure 14. Number of compactions in progress

Figure 15 shows the exploratory spatio-temporal visualization of the compaction works in progress.

Furthermore, Figure 16 shows the progress curve of the compaction works.

The compaction works by the vibration roller are done intermittently. As reported in Section 4.1, the concrete haulage still reserves the capacity. So, it is possible to increase more the concrete placement areas in parallel. In contrast, earthworks volume per hour burden on a backhoe would be larger.

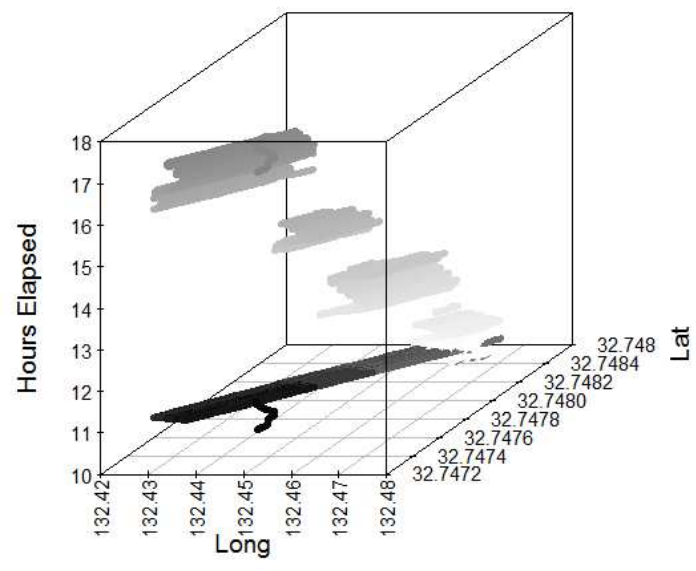

Figure 15. Exploratory spatio-temporal visualization of compaction works in progress

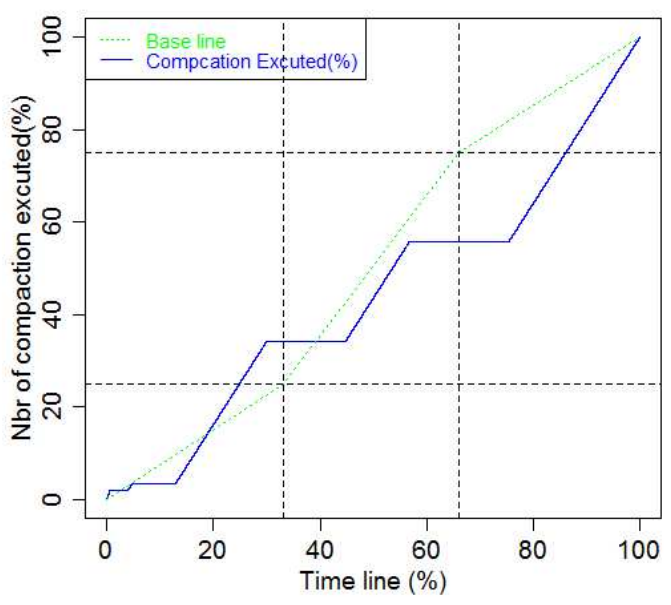

Figure 16. Progress curve of compaction works

\section{Conclusion and Some Open Problems}

The information modelling presented in this paper means a process including:

(1) Automatically gathering field data,

(2) Feedback of physical cues as to machine behaviour,

(3) Showing exploratory spatio-temporal visualization of hazards latent in in-situ earthwork process, and 
(4) Enhancement of communication regarding work process and operations.

And then the information modelling system enables workers to:

(1) Pick a set of building block of information at the right level of abstraction,

(2) Provide operators with the opportunities to reflect on their own bearings as facing their own works at hand,

(3) Reduce likely stress in remote control operations of machines, and

(4) Take timely and quickly correct actions based on detailed visibility of appearances and motions of the mobile entities in a whole unmanned construction process.

Taken together, the workers concerned could get indepth insight into the work process while showing hints to improve safety and productivity with detailed reporting. Accordingly, it would be possible to secure traceability and accountability with the mechanized earthworks.

In future, remotely dexterous operations in close proximity to other would be required, when unmanned construction system would be deployed in various kinds of post-disaster recovery and reconstruction, for example, approaching to the target (e.g., inspection, repair, monitoring another one), positioning or repositioning dangerous materials, connecting an object with other, grasping and stacking sandbags or gabions, inserting nozzle into a fuel filler port for replenishment, and so on. For securing safety, reliable and effective operations, we will be not just focusing on the best way but the variety of better ways to provide operators with physical cues, which would enhance

(1) Awakening spatial awareness,

(2) Perception of distance and clearance,

(3) Recognition of possible motion space,

(4) Grasping posture of machine body, and

(5) Improvement of operator's skill.

In addition, we will do further research and development on exploratory spatio-temporal visualization of:

(1) Earthworks by backhoe,

(2) Terrain being changed with earthwork operations, and

(3) Civil engineering structure under construction

\section{References}

[1] Adalsteinsson D. H. GPS machine guidance in construction equipment, on-line:

http://www.trimble-

productivity.com/media/pdf/ProductivityReportGP ForExcavators2008.pdf

[2] MALAGA Demonstration \& Learning Center
Road Construction Production Study on-line: http://www.trimble-

productivity.com/media/pdf/ProductivityReportCA TRoadConstruction2006.pdf

[3] Open Source Initiative: GNU General Public License, open line:

http://opensource.org/licenses/gpl-2.0.php

[4] Andrienko N., Andrienko G., Gatalsky P. Exploratory spatio-temporal visualization: an analytical review, Journal of Visual Languages and Computing \& Computing, 14: 503-541, 2003.

[5] Blok C. Monitoring change: characteristics of dynamic geo-spatial phenomena for visual exploration, in: Ch. Freksa et al. (Eds.), Spatial Cognition II, Lecture Notes in Artificial Intelligence, Vol. 1849, pages 16-30, Springer, Berlin, Heidelberg, 2000.

[6] The R Project for Statistical Computing, on-line: http://www.r-project.org/

[7] Nishigaki S., Saibara K., Kitahara S., Matsuda F., et al. LHD Remote Monitoring and Management based on Delay/Disruption Tolerant Network, in Proceeding of the 27 $7^{\text {th }}$ ISARC, pages 326-335, Bratislava, Slovakia, 2010

[8] Nishigaki S., Saibara K., Kitahara S., Fujino K. Quantative Indexes to Evaluate Operator's Bearing, Difficulty in Task, and Machine Operability when Remotely Controlling Backhoe, In Proceeding of the $30^{\text {th }}$ ISARC, Montreal, Canada, 2013.

[9] Nishigaki S., Saibara K., Kitahara S., Iwasaki H., Yamada K., Satoh H. ICT-based work management to support unmanned construction for post-disaster, In Proceedings of the $28^{\text {th }}$ ISARC, pages 508-513, Seoul, Korea, 2011.

[10] Saibara K., Nishigaki S., Matsuda F. Network communication note to assist backhoe operators and dump truck drivers for earthwork, In Proceedings of the $30^{\text {th }}$ ISARC, Crane Operation in Construction Projects, 257, Montreal, Canada, 2013.

[11] Nishigaki1 S., Sibara K., Kitahara S., et al. Network Communication Note and Visualization of Work Process to Enhance Unmanned Construction, ICCBEI 2013 